\title{
Cooperative effect of cell-cycle regulators expression on bladder cancer development and biologic aggressiveness
}

\author{
Shahrokh F Shariat ${ }^{1}$, Alexandre R Zlotta ${ }^{2}$, Raheela Ashfaq ${ }^{3}$, Arthur I Sagalowsky ${ }^{1}$ and \\ Yair Lotan ${ }^{1}$ \\ ${ }^{1}$ Department of Urology, University of Texas Southwestern Medical Center, Dallas, TX, USA; ${ }^{2}$ Department \\ of Urology, Mount Sinai and Princess Margaret Hospitals, University of Toronto, Toronto, ON, Canada \\ and ${ }^{3}$ Department of Pathology, University of Texas Southwestern Medical Center, Dallas, TX, USA
}

\begin{abstract}
The aim of this study was to evaluate the association between p53, p21, p27 and Rb expression, alone or in combination, with pathological features and clinical outcomes of urothelial carcinoma of the bladder. Immunohistochemical staining for p53, p21, p27 and pRB was performed on tissue microarrays comprising normal urothelium from nine controls, transurethral resection specimens from 74 patients with Ta, Tis and/or T1 bladder urothelial carcinoma, radical cystectomy specimens from 226 consecutive urothelial carcinoma patients, and lymph nodes with tumor invasion from 50 of the 226 cystectomy patients. All nine controls had normal status of biomarkers. The proportion of biomarkers alterations was highest in lymph node metastases. p53, pRB and p27 were associated with pathologic stage, lymphovascular invasion and lymph node metastases $(P$-values $\leq 0.050)$. Each biomarker alone, each combination of two biomarkers and the number of altered biomarkers were all independently associated with disease recurrence and bladder cancer-specific death $(P$ values $\leq \mathbf{0 . 0 4 6}$ ). In patients with organ-confined disease, p53, p21 and p27 were independently associated with disease recurrence and bladder cancer-specific death $(P$-values $\leq 0.046)$. There was a good but not perfect concordance in the expression of biomarkers between matched transurethral resection and cystectomy specimens ( $n=22$; concordance rates: $77-86 \%)$ and between matched lymph node and cystectomy specimens $(n=50,70-92 \%)$. In conclusion, p53, p21, p27 and pRB have a cooperative/synergistic role in the biologic behavior of bladder urothelial carcinoma. In contrast to the use of single biological markers, evaluation of their combined status may improve prognostic models and help identify patients who might benefit from adjuvant therapies and in decision-making.
\end{abstract}

Modern Pathology (2007) 20, 445-459. doi:10.1038/modpathol.3800757; published online 2 March 2007

Keywords: p53; p21; p27; retinoblastoma; progression; bladder cancer

Elucidation of molecular pathways involved in urothelial carcinoma of the bladder is essential for our understanding of carcinogenesis and disease progression. Mutations of cell cycle regulatory genes are the genetic alterations most commonly found in human neoplasia, including bladder cancer. ${ }^{1-4} \mathrm{Al}-$ terations in the $p 53$ and retinoblastoma $(R B)$ tumor suppressor genes have been shown to play an important role in the development of urothelial carcinoma. However, downstream pathways that contribute to urothelial transformation are not

Correspondence: Dr SF Shariat, MD, Department of Urology, University of Texas Southwestern Medical Center, 5323 Harry Hines Boulevard, Dallas, TX 75390-9110, USA.

E-mail: Shahrokh.Shariat@UTSouthwestern.edu

Received 02 October 2006; revised 09 January 2007; accepted 16 January 2007; published online 2 March 2007 completely defined and are key factors too. ${ }^{5,6}$ Members of the kinase inhibitor protein (KIP) family, p21 ${ }^{\mathrm{WAF} 1 / \mathrm{CIP} 1}$ and p27 ${ }^{\mathrm{Kip} 1}$ are both p53-inducible and p53-independent cyclin-dependent kinase inhibitors that can arrest the cell by inhibiting DNA replication. Alteration of p53, pRB, p21 and p27 expression has prognostic significance in patients treated with radical cystectomy for bladder cancer. ${ }^{5,7-14}$ Combinations of independent, complementary markers may provide a more accurate prediction of outcome compared to a single marker. ${ }^{5,13-16}$

A problem with the measurement of markers is the possible discordance between immunohistochemistry and biological function. This is especially true as multiple redundant pathways might exist to stimulate downstream effectors. This discrepancy between expression and function and the demon- 
stration of alternate or redundant pathways might provide an explanation for the inconsistent results found in the literature about the use of single biological markers as prognostic factors in urothelial carcinoma.

The aims of the present study were to correlate the expression of p53, p21, p27 and pRB, alone or in combination, with urothelial carcinoma presence, clinical and pathologic characteristics. Towards this end, we assessed the differential expression of $\mathrm{p} 53$, p21, p27 and pRB in (1) normal bladder urothelium; (2) urothelial carcinoma tissue from patients treated with transurethral resection for Ta, Tis and/or T1; (3) urothelial carcinoma tissue from patients treated with radical cystectomy for invasive disease; (4) concomitant carcinoma in-situ areas within the cystectomy specimens; (5) histologically normal urothelium within the cystectomy specimens; and (6) malignant lymph node tissue involved with urothelial carcinoma. In addition, this study aimed to correlate p53, p21, p27 and pRB expression between radical cystectomy and matched transurethral resection specimens from the same patients and between radical cystectomy and matched metastatic lymph node specimens.

\section{Materials and methods}

\section{Patient Population}

All studies were undertaken with the approval and institutional oversight of the Institutional Review Board for the Protection of Human Subjects at University of Texas Southwestern Medical School. The study comprised three patient cohorts: 226 patients who underwent radical cystectomy with bilateral lymphadenectomy, 74 patients who underwent transurethral resection and nine patients who underwent cystectomy for other causes than malignancy without evidence of any genitourinary malignancy.

All 383 consecutive patients admitted at our institution for treatment of urothelial carcinoma with radical cystectomy and bilateral lymphadenectomy during the period from January/1987 to December/2002 were potential candidates for these analyses. Our analyses were limited to patients who had urothelial carcinoma. Adequate archival tissue was available for 226 of the 383 cystectomy patients. Histology, grade, stage and presence of carcinoma in situ were confirmed by blinded review of the original pathology slides. The indications for radical cystectomy were tumor invasion into the muscularis propria or prostastic stroma or T1/carcinoma in-situ refractory to transurethral resection with intravesical chemotherapy and/or immunotherapy. No patient had distant metastatic disease at the time of cystectomy. For each patient, comprehensive clinical and pathologic data elements were collected and entered into an institutional review boardapproved database. Multiple data reviews and quality checks were performed to assure the accuracy and completeness of data elements. Chemotherapy regimens consisted of methotrexate, vinblastine, doxorubicin and cisplatin. Sixty of the 226 patients $(26.5 \%)$ underwent adjuvant chemotherapy for positive lymph node status and/or extra-vesical extension.

Our study also comprised a convenience sample of 74 patients, who underwent transurethral resection at our institution for treatment of Ta, Tis and/or T1 during the period from 2/1995 to $12 / 2003$. Sixtythree patients $(85 \%)$ underwent previous transurethral resection. Fifty-four patients $(73 \%)$ were treated with intravesical therapy including Bacillus Calmette-Guerin and Mitomycin C. Twenty-two patients $(30 \%)$ ultimately underwent radical cystectomy for persistent disease, cancer progression or intolerance of intravesical therapy. Median age at transurethral resection was 63 years (range: 41-89).

\section{Immunohistochemistry and Scoring}

We performed p53, p21, pRB and p27 immunohistochemical staining using serial sections from the same paraffin-embedded tissue microarray blocks (see Appendix A for tissue microarray construction). ${ }^{13}$ Staining and scoring protocols for p53 (monoclonal mouse anti-human antibody DO-7; Dako, Carpinteria, CA; dilution 1:4000), ${ }^{17}$ p21 (monoclonal mouse anti-human, SX118, Dako; dilution 1:200), ${ }^{16}$ pRB (monoclonal mouse anti-human; RB1, Dako; dilution 1:75) ${ }^{13}$ and p27 (monoclonal mouse anti-human, SX53G8; Dako; dilution 1:400) ${ }^{18}$ have been described elsewhere (see Appendix B for detailed protocols). Immunostaining was performed on the Dako Autostainer (Carpinteria, CA). Optimum primary antibody dilutions were predetermined using known positive control tissues. Multiple known, established positive control sections were included in each run. Tumor sections with the primary antibodies substituted with rabbit immunoglobulin fraction (Normal) and/or IgG 1 monoclonal were used as negative controls.

We used bright-field microscopy imaging coupled with advanced color detection software (Automated Cellular Imaging System, ChromaVision Medical Systems Inc., San Juan Capistrano, CA) to detect, classify, and counter stain cellular objects based on predetermined color morphology. We obtained the mean, maximum, range and standard deviation of staining intensity and percent positive nuclei/area measurements by using 10 random hot spots within each specimen. The mean of the triplicate cores was calculated for data analysis.

All markers were placed in one of two categories, altered or normal.,11,13,18,19 Nuclear p53 immunoreactivity was considered altered when samples demonstrated at least $10 \%$ nuclear reactivity. ${ }^{4,19}$ p21 immunoreactivity was considered altered when samples demonstrated no detectable or only very 
low levels of p21 nuclear staining. Tumors with no pRB expression and those with a strong homogeneous staining pattern (more than 50\%) were categorized as having altered pRB status. ${ }^{11,13}$ Nuclear p27 immunoreactivity was considered altered when samples demonstrated less than $30 \%$ nuclear reactivity. ${ }^{18}$

\section{Pathology}

Staff pathologists with expertise in genitourinary pathology examined all specimens according to previously published protocols. ${ }^{20}$ The 2002 TNM classification was used for pathologic staging, and the 1973 WHO classification for pathologic grading. To ensure validity of the data extraction, two clinicians read pathology reports of 162 consecutive cystectomy patients, whereas blinded to patient clinical parameters and the finding of the other reviewer. Inter-reader reliability measured using the intra-class correlation coefficient was greater than 0.95 for all pathologic parameters.

\section{Follow-up}

Patients generally were seen post-operatively at least every 3-4 months for the first year, semiannually for the second year, and annually thereafter. Follow-up visits generally consisted of a physical examination, serum chemistry evaluation, along with liver function tests and alkaline phosphatase when clinically indicated. Diagnostic imaging of the upper tracts such as ultrasonography and/or intravenous pyelography and chest radiography were performed at least annually or when clinically indicated. Bone scan and/or computerized tomography were performed at the discretion of the treating physician. Detection of cancer in the ureter and/or urethra was coded as second primaries and not as local or distant recurrence. When patients died, the cause of death was determined by the treating physicians, by chart review corroborated by death certificates or by death certificates alone. Most patients who were identified as having died of bladder cancer had progressive, widely disseminated and often highly symptomatic metastases at the time of death. Peri-operative mortality (death within 30 days of surgery) was censored at time of death for bladder cancer-specific survival analyses.

\section{Statistical Analysis}

The Fisher's exact test and the $\chi^{2}$ test were used to evaluate the association between molecular markers and clinico-pathologic parameters. Differences in variables with a continuous distribution across dichotomous or ranked categories were assessed using the Mann-Whitney U-test or the KruskalWallis nonparametric analysis of variance, respec- tively. The Kaplan-Meier method was used to calculate survival functions, and differences were assessed with the log rank statistic. Univariable and multivariable survival analyses were performed using the Cox proportional hazard regression model. The lowest category was used as the referent category when calculating the hazards ratios. Statistical significance in this study was set as $P \leq 0.050$. All reported $P$-values are two-sided. All analyses were performed with SPSS (version 13.0).

\section{Results}

\section{Expression of p53, pRB, p21 and p27}

The differential expression of the four biomarkers in different tissues at various stages of bladder cancer is shown in Table 1. For all four biomarkers, the prevalence of alteration incrementally increased from normal urothelium to metastatic lymph nodes. Normal bladder urothelium from all nine control patients showed normal status of pRB (heterogeneous), p53 (absent), p21 (positive) and p27 (positive). p53 and pRB staining patterns were exclusively nuclear. Strong expression of p27 was consistently noted in nuclei of normal urothelium, and there was a consistent but variable reduction in the number of p27-stained nuclei in tumor tissue. Although normal cases showed homogeneous immunostaining, cancer cases exhibited a heterogeneous pattern of significantly reduced p27 immunoreactivity. In most cases, staining was confined to the nucleus.

\section{Association of p53, pRB, p21 and p27 as Individual Variables with Pathologic Characteristics}

Molecular and pathologic characteristics of the 226 radical cystectomy patients and association with p53, pRB, p21 and p27 expression are shown in Table 2. The expression of p53, pRB and p27 were significantly associated with pathologic tumor stage, lymphovascular invasion, lymph node metastases and stage grouping $(<\mathrm{pT} 3$ No vs $\geq$ T3 No vs pTany Npositive). p53 was also significantly associated with pathologic tumor grade and presence of concomitant carcinoma in situ.

Median age at radical cystectomy was 66 years (range: 34-88). Patients with altered p53, altered pRB or altered p27 had significantly higher numbers of metastatic lymph nodes and percent metastatic lymph nodes compared with patients with normal status of these markers.

There was a statistically significant concordance (both markers altered or normal) between p53 and pRB (concordance rate $=61 \%, P=0.001$ ), p53 and p27 (concordance rate $=59 \%, P=0.010$ ) and pRB and p27 (concordance rate $=61 \%, P=0.001$ ). The concordance between p53 and p21 (concordance rate $=52 \%, P=0.326$ ), between $\mathrm{pRB}$ and $\mathrm{p} 21$ (con- 
Table 1 Differential expression of p53, pRB, p21, p27, and number of altered biomarkers in subjects with and without urothelial cell carcinoma of the bladder

\begin{tabular}{|c|c|c|c|c|c|c|c|c|c|c|}
\hline & \multicolumn{5}{|c|}{ Individual biomarkers } & \multicolumn{5}{|c|}{ Number of altered biomarkers } \\
\hline & $\begin{array}{c}\text { No. of } \\
\text { patients }\end{array}$ & $\begin{array}{l}\text { Altered } \\
\text { p53 }\end{array}$ & $\begin{array}{l}\text { Altered } \\
\quad p R B\end{array}$ & $\begin{array}{l}\text { Altered } \\
\text { p21 }\end{array}$ & $\begin{array}{l}\text { Altered } \\
\text { p27 }\end{array}$ & None & One & Two & Three & Four \\
\hline $\begin{array}{l}\text { Normal urothelium } \\
\text { from subjects without } \\
\text { bladder TCC }\end{array}$ & 9 & 0 & 0 & 0 & 0 & 9 & 0 & 0 & 0 & \\
\hline $\begin{array}{l}\text { TURBT specimens from } \\
\text { subjects with Ta, Tis, } \\
\text { and/or T1 TCC }\end{array}$ & 74 & $25(34 \%)$ & $29(39 \%)$ & $26(35 \%)$ & $35(47 \%)$ & $16(21.6)$ & $23(31 \%)$ & $15(20 \%)$ & $17(23 \%)$ & $3(4 \%)$ \\
\hline \multicolumn{11}{|c|}{ Cystectomy specimens from subjects treated with radical cystectomy } \\
\hline Primary, index TCC & 226 & $120(53 \%)$ & $112(50 \%)$ & $76(34 \%)$ & $136(60 \%)$ & $28(12 \%)$ & $56(25 \%)$ & $59(26 \%)$ & $62(27 \%)$ & $21(9 \%)$ \\
\hline $\begin{array}{l}\text { Histologically normal } \\
\text { urothelium adjacent to } \\
\text { primary TCC }\end{array}$ & 72 & $11(15 \%)$ & $16(22 \%)$ & $16(22 \%)$ & $19(26 \%)$ & $25(35 \%)$ & $34(47 \%)$ & $11(15 \%)$ & $2(3 \%)$ & $0(0 \%)$ \\
\hline $\begin{array}{l}\text { Concomitant carcinoma } \\
\text { in situ areas }\end{array}$ & 40 & $18(45 \%)$ & $17(43 \%)$ & $14(35 \%)$ & $21(53 \%)$ & $4(10 \%)$ & $14(35 \%)$ & $14(35 \%)$ & $6(15 \%)$ & $2(5 \%)$ \\
\hline $\begin{array}{l}\text { Metastatic lymphoid } \\
\text { tissue from subjects } \\
\text { treated with radical } \\
\text { cystectomy }\end{array}$ & 50 & $38(76 \%)$ & $34(68 \%)$ & $23(46 \%)$ & $32(64 \%)$ & $1(2 \%)$ & $8(16 \%)$ & $12(24 \%)$ & $21(42 \%)$ & $8(16 \%)$ \\
\hline
\end{tabular}

Abbreviations: TCC, transitional cell carcinoma; TURBT, transurethral resection of bladder tumor.

cordance rate $=55 \%, P=0.159)$ and between p21 and p27 (concordance rate $=50 \%, P=0.251$ ) were not statistically significant.

\section{Association of p53, pRB, p21 and p27 as Individual Variables with Clinical Outcomes}

Disease recurred in 101/226 patients (45\%) and 100/ 226 (44\%) patients were dead at the time of analysis. Of these 100 patients, 82 patients died of metastatic bladder cancer $(82 \%)$ and 18 patients died of other causes without evidence of disease progression $(18 \%)$. The mean follow-up was 51 months (median 37 , range 2-183) for those patients alive at the time of analysis. Kaplan-Meier analyses (Table 3) showed that altered expression of p53, pRB, p21 and p27 were each significantly associated with an increased probability of disease recurrence and bladder cancer-specific mortality.

In multivariable Cox proportional hazards regression analyses that adjusted for the effects of pathologic stage, tumor grade, lymphovascular invasion, adjuvant chemotherapy, lymph node status and one of the four biomarkers, each biomarker was independently associated with disease recurrence $(P$-values $\leq 0.039)$ and bladder cancer-specific mortality $(P$-values $\leq 0.041)$.

In the subgroup of patients with organ-confined disease $(<\mathrm{pT} 3 ; n=97,43 \%), \mathrm{p} 53, \mathrm{p} 21$ and p27, but not $\mathrm{pRB}$, were independently associated with disease recurrence $(P$-values $\leq 0.042)$ and bladder cancer-specific mortality $(P$-values $\leq 0.046)$ after adjusting for the effects of pathologic stage (nonmuscle invasive versus muscle invasive stage), pathologic grade, adjuvant chemotherapy, lymphovascular invasion and lymph node status.

\section{Association of p53, pRB, p21 and p27 as Combined Variables with Pathologic Characteristics and Clinical Outcomes}

We repeated the analyses using six possible combinations of biomarkers: p53 and pRB, p53 and p21, p53 and p27, pRB and p21, pRB and p27 and p21 and $\mathrm{p} 27$. We created three categories within each combination: both normal, one altered and one normal and both altered. The association of the combined biomarkers with clinical and pathologic characteristics is shown in Table 4.

Kaplan-Meier analysis demonstrated that patients could be stratified into statistically significantly different risk groups for disease recurrence and bladder cancer-specific mortality based on the combined status of each combination (Table 3; Figures 1 and 2).

The combined biomarkers were then analyzed in separate multivariable Cox proportional hazards regression analyses that adjusted for effects of standard variables. Each combination was independently associated with disease recurrence ( $P$-values $\leq 0.031)$ and bladder cancer-specific mortality $(P$-values $\leq 0.038)$. A representative multivariable model is shown in Table 5 (Model 1). The statistical significance of these analyses was retained when 
Table 2 Associations of molecular markers as individual variables with clinical and pathologic characteristics in 226 consecutive patients treated with radical cystectomy for urothelial cell carcinoma of the bladder

\begin{tabular}{|c|c|c|c|c|c|c|c|c|c|}
\hline & \multirow[b]{2}{*}{ No. of patients (\%) } & \multicolumn{2}{|c|}{ p53 expression } & \multicolumn{2}{|c|}{$p R B$ expression } & \multicolumn{2}{|c|}{ p21 expression } & \multicolumn{2}{|c|}{ p27 expression } \\
\hline & & Altered & $\mathrm{P}$ & Altered & $\mathrm{P}$ & Altered & $\mathrm{P}$ & Altered & $\mathrm{P}$ \\
\hline Total (\%) & $226(100)$ & $120(53)$ & & $112(50)$ & & $76(34)$ & & $136(60)$ & \\
\hline \multicolumn{10}{|l|}{ Gender (\%) } \\
\hline Female & $46(20)$ & $25(54)$ & & $26(57)$ & & $15(33)$ & & $34(74)$ & \\
\hline Male & $180(80)$ & $95(53)$ & 0.870 & $86(48)$ & 0.324 & $61(34)$ & 1.000 & $102(57)$ & 0.042 \\
\hline \multicolumn{10}{|l|}{ Pathologic stage (\%) } \\
\hline pTa, pTis or pT1 & $42(19)$ & $14(33)$ & & $15(36)$ & & $12(29)$ & & $19(45)$ & \\
\hline pT2 & $65(29)$ & $27(42)$ & & $26(40)$ & & $17(26)$ & & $32(49)$ & \\
\hline pT3 & $83(37)$ & $54(65)$ & & $48(58)$ & & $29(35)$ & & $60(72)$ & \\
\hline pT4 & $36(16)$ & $25(69)$ & $<0.001$ & $23(64)$ & 0.013 & $18(50)$ & 0.090 & $25(69)$ & 0.004 \\
\hline \multicolumn{10}{|l|}{ Pathological grade (\%) } \\
\hline Grade 1 or 2 & $17(8)$ & $2(12)$ & & $7(41)$ & & $5(29)$ & & $8(47)$ & \\
\hline Grade 3 & $209(92)$ & $118(57)$ & 0.001 & $105(50)$ & 0.615 & $71(34)$ & 0.795 & $128(61)$ & 0.305 \\
\hline \multicolumn{10}{|l|}{ Lymph node status (\%) ${ }^{\mathrm{a}}$} \\
\hline No & $160(71)$ & $67(42)$ & & $72(45)$ & & $51(32)$ & & $85(53)$ & \\
\hline $\mathrm{N} 1, \mathrm{~N} 2$ & $65(29)$ & $52(80)$ & $<0.001$ & $39(60)$ & 0.050 & $25(39)$ & 0.355 & $50(77)$ & 0.001 \\
\hline \multicolumn{10}{|l|}{ Lymphovascular invasion (\%) } \\
\hline Negative & $124(55)$ & $49(40)$ & & $52(42)$ & & $38(31)$ & & $65(52)$ & \\
\hline Positive & $101(45)$ & $70(69)$ & $<0.001$ & $59(58)$ & 0.016 & $38(38)$ & 0.321 & $70(69)$ & 0.014 \\
\hline \multicolumn{10}{|l|}{ Concomitant CIS (\%) ${ }^{\mathrm{a}}$} \\
\hline Negative & $132(59)$ & $61(46)$ & & $65(49)$ & & $45(34)$ & & $75(57)$ & \\
\hline Positive & $93(42)$ & $58(62)$ & 0.021 & $46(50)$ & 1.000 & $31(33)$ & 1.000 & $60(65)$ & 0.271 \\
\hline \multicolumn{10}{|l|}{ Stage grouping ${ }^{\mathrm{a}}$} \\
\hline Organ confined (<pT3 No) & $97(43)$ & $32(33)$ & & $36(37)$ & & $25(25)$ & & $45(46)$ & \\
\hline Extravesical extension (pT3 No) & $64(28)$ & $36(56)$ & & $37(58)$ & & $26(41)$ & & $41(64)$ & \\
\hline $\begin{array}{l}\text { Lymph node metastases } \\
\text { (pTany N positive) }\end{array}$ & $65(29)$ & $52(80)$ & $<0.001$ & $39(60)$ & 0.005 & $25(39)$ & 0.092 & $50(77)$ & $<0.001$ \\
\hline \multicolumn{10}{|l|}{ P53 expression (\%) } \\
\hline Wild type & $106(47)$ & - & & $40(38)$ & & $32(30)$ & & $54(51)$ & \\
\hline Altered & $120(53)$ & - & - & $72(60)$ & 0.001 & $44(37)$ & 0.326 & $82(68)$ & 0.010 \\
\hline \multicolumn{10}{|l|}{ pRB expression (\%) } \\
\hline Wild type & $114(50)$ & $48(42)$ & & - & & $33(29)$ & & $56(49)$ & \\
\hline Altered & $112(50)$ & $72(64)$ & 0.001 & - & - & $43(38)$ & 0.159 & $80(71)$ & 0.001 \\
\hline \multicolumn{10}{|l|}{ P21 expression (\%) } \\
\hline Wild type & $150(66)$ & $76(51)$ & & $69(46)$ & & - & & $86(57)$ & \\
\hline Altered & $76(34)$ & $44(58)$ & 0.326 & $43(57)$ & 0.159 & - & - & $50(66)$ & 0.251 \\
\hline
\end{tabular}

Abbreviation: CIS = carcinoma in situ.

${ }^{\mathrm{a}}$ Lymph node status, lymphovascular invasion, and concomitant carcinoma in situ were not available in one patient.

performed in patients with organ-confined disease (data not shown).

\section{Association of the Number of Altered Markers as Ranked Variable with Pathologic Characteristics and Clinical Outcomes}

Twenty-eight of the 226 patients (12\%) exhibited normal status of all markers; 56/226 tumors (25\%) exhibited alteration of one biomarker; 59/226 tumors $(26 \%)$ exhibited alteration of two biomarkers, $62 / 226$ tumors $(27 \%)$ exhibited alterations of three biomarkers and 21/226 tumors (9\%) exhibited alterations of all four biomarkers.

The association of the number of altered biomarkers with clinical and pathologic characteristics of the 226 radical cystectomy patients is shown in Table 6. The number of altered biomarkers increased with advancing pathologic tumor stage $(P<0.001)$, higher rate of lymphovascular invasion $(P<0.001)$, higher rate of lymph node metastases $(P<0.001)$ and more adverse stage grouping $(P<0.001)$ (Figures 1 and 2).

Kaplan-Meier analyses (Table 3, Figure 3) showed that the risk of disease recurrence $(P$-for 
Table 3 Univariate associations of molecular markers as individual and combined variables with recurrence-free survival and bladder cancer-specific survival in 226 consecutive patients treated with radical cystectomy for urothelial cell carcinoma of the bladder

\begin{tabular}{|c|c|c|c|c|c|c|}
\hline & \multicolumn{3}{|c|}{ Recurrence-free survival estimates (\%) } & \multicolumn{3}{|c|}{ Bladder cancer-specific survival estimates (\%) } \\
\hline & 5-year \pm s.e. & $10-$ year \pm s.e. & $\mathrm{P}^{\mathrm{a}}$ & 5 -year \pm s.e. & 10-year \pm s.e. & $\mathrm{P}^{\mathrm{a}}$ \\
\hline \multicolumn{7}{|l|}{ p53 } \\
\hline Wt & $75.0 \pm 4.6$ & $63.0 \pm 6.5$ & & $77.9 \pm 4.7$ & $70.1 \pm 6.1$ & \\
\hline Alt & $42.5 \pm 5.4$ & $36.1 \pm 5.8$ & $<0.001$ & $42.5 \pm 5.3$ & $29.1 \pm 6.9$ & $<0.001$ \\
\hline \multicolumn{7}{|l|}{$p 21$} \\
\hline $\mathrm{Wt}$ & $64.0 \pm 4.7$ & $57.0 \pm 5.7$ & & $70.3 \pm 4.3$ & $55.9 \pm 7.7$ & \\
\hline Alt & $35.8 \pm 6.6$ & $35.8 \pm 6.6$ & $<0.001$ & $37.2 \pm 6.5$ & $32.6 \pm 7.1$ & $<0.001$ \\
\hline \multicolumn{7}{|l|}{ p27 } \\
\hline $\mathrm{Wt}$ & $75.6 \pm 5.4$ & $75.6 \pm 5.4$ & & $68.9 \pm 5.6$ & $64.0 \pm 7.0$ & \\
\hline Alt & $41.6 \pm 5.2$ & $34.1 \pm 5.8$ & $<0.001$ & $51.6 \pm 5.1$ & $35.8 \pm 8.1$ & 0.019 \\
\hline \multicolumn{7}{|l|}{$p R B$} \\
\hline Wt & $64.0 \pm 5.1$ & $60.6 \pm 5.8$ & & $71.5 \pm 4.9$ & $61.3 \pm 10.3$ & \\
\hline Alt & $43.6 \pm 5.9$ & $38.3 \pm 6.3$ & 0.020 & $45.4 \pm 5.5$ & $31.8 \pm 6.6$ & $<0.001$ \\
\hline \multicolumn{7}{|l|}{ p53 and p21 } \\
\hline $\mathrm{Wt}$ & $80.6 \pm 5.1$ & $65.7 \pm 8.2$ & & $87.1 \pm 4.8$ & $78.7 \pm 7.2$ & \\
\hline Any one alt & $53.1 \pm 5.7$ & $50.3 \pm 6.1$ & & $55.1 \pm 5.4$ & $41.7 \pm 7.8$ & \\
\hline Both alt & $19.6 \pm 7.5$ & $19.6 \pm 7.5$ & $<0.001$ & $25.1 \pm 7.8$ & $16.8 \pm 8.6$ & $<0.001$ \\
\hline \multicolumn{7}{|l|}{ p53 and p27 } \\
\hline $\mathrm{Wt}$ & $81.2 \pm 5.7$ & $81.2 \pm 5.7$ & & $77.2 \pm 6.5$ & $77.2 \pm 6.5$ & \\
\hline Any one alt & $56.1 \pm 6.9$ & $45.6 \pm 8.9$ & & $66.4 \pm 6.3$ & $49.6 \pm 10.3$ & \\
\hline Both alt & $30.6 \pm 6.1$ & $27.2 \pm 6.3$ & $<0.001$ & $37.1 \pm 6.1$ & $24.9 \pm 7.6$ & $<0.001$ \\
\hline \multicolumn{7}{|l|}{$p 53$ and $p R B$} \\
\hline Wt & $78.0 \pm 5.8$ & $73.1 \pm 7.2$ & & $84.5 \pm 5.4$ & $84.5 \pm 5.4$ & \\
\hline Any one alt & $48.3 \pm 6.5$ & $44.3 \pm 7.1$ & & $56.4 \pm 6.2$ & $32.3 \pm 6.3$ & \\
\hline Both alt & $41.9 \pm 7.3$ & $32.8 \pm 7.5$ & $<0.001$ & $36.2 \pm 6.5$ & $25.6 \pm 7.0$ & $<0.001$ \\
\hline \multicolumn{7}{|l|}{$p R B$ and $p 21$} \\
\hline Wt & $76.1 \pm 5.1$ & $68.3 \pm 7.2$ & & $86.1 \pm 14.2$ & $68.9 \pm 15.8$ & \\
\hline Any one alt & $46.7 \pm 6.1$ & $41.6 \pm 6.4$ & & $48.3 \pm 5.8$ & $39.6 \pm 6.2$ & \\
\hline Both alt & $32.9 \pm 8.9$ & $32.9 \pm 8.9$ & $<0.001$ & $35.0 \pm 8.4$ & $17.5 \pm 13.1$ & $<0.001$ \\
\hline \multicolumn{7}{|l|}{$p 21$ and $p 27$} \\
\hline Wt & $82.8 \pm 5.3$ & $82.8 \pm 5.3$ & & $76.4 \pm 6.2$ & $76.4 \pm 6.2$ & \\
\hline Any one alt & $49.3 \pm 5.8$ & $41.4 \pm 6.4$ & & $62.0 \pm 5.2$ & $38.9 \pm 10.3$ & \\
\hline Both alt & $29.0 \pm 7.7$ & $29.0 \pm 7.7$ & $<0.001$ & $29.8 \pm 7.7$ & $29.8 \pm 7.7$ & $<0.001$ \\
\hline \multicolumn{7}{|l|}{$p R B$ and $p 27$} \\
\hline $\mathrm{Wt}$ & $81.3 \pm 5.4$ & $81.3 \pm 5.4$ & & $82.3 \pm 5.4$ & $82.3 \pm 5.4$ & \\
\hline Any one alt & $48.4 \pm 6.4$ & $43.5 \pm 7.3$ & & $58.3 \pm 5.9$ & $38.2 \pm 12.3$ & \\
\hline Both alt & $39.4 \pm 6.9$ & $32.2 \pm 7.3$ & $<0.001$ & $44.6 \pm 6.6$ & $30.9 \pm 7.4$ & $<0.001$ \\
\hline \multicolumn{7}{|c|}{ Number of alterations } \\
\hline None & $96.3 \pm 3.6$ & $96.3 \pm 3.6$ & & $96.3 \pm 3.6$ & $96.3 \pm 3.6$ & \\
\hline One & $80.3 \pm 6.1$ & $80.3 \pm 6.1$ & & $67.9 \pm 7.3$ & $61.1 \pm 9.2$ & \\
\hline Two & $51.7 \pm 7.8$ & $30.5 \pm 13.0$ & & $51.1 \pm 8.2$ & $45.4 \pm 9.0$ & \\
\hline Three & $44.6 \pm 7.0$ & $29.8 \pm 8.6$ & & $33.5 \pm 7.2$ & $28.7 \pm 7.6$ & \\
\hline Four & $17.0 \pm 10.0$ & $17.0 \pm 10.0$ & $<0.001$ & $22.9 \pm 11.3$ & $22.9 \pm 11.3$ & $<0.001$ \\
\hline
\end{tabular}

Abbreviations: alt, altered; wt, wild type.

${ }^{\mathrm{a}}$ Log rank statistics.

trend $<0.001)$ and bladder cancer-specific mortality $(P$-for trend $<0.001)$ increased with the number of altered biomarkers. In a multivariable Cox proportional hazards regression analysis (Table 5, model 2) that adjusted for the effects of standard variables, increasing number of altered biomarkers was in- dependently associated with higher risk of disease recurrence $(P$-for trend $=0.042)$ and bladder cancerspecific mortality $(P$-for trend $=0.046)$. The statistical significance of these analyses was retained when performed in patients with organ-confined disease (data not shown). 
Table 4 Associations of combined biomarkers with clinical and pathologic characteristics in 226 consecutive patients treated with radical cystectomy for urothelial cell carcinoma of the bladder

\begin{tabular}{|c|c|c|c|c|c|c|c|c|c|}
\hline & \multicolumn{3}{|c|}{ p53 and pRB expression } & \multicolumn{3}{|c|}{ p53 and p21 expression } & \multicolumn{3}{|c|}{ p53 and p27 expression } \\
\hline & One altered & Both altered & $\mathrm{P}$ & One altered & Both altered & $\mathrm{P}$ & One altered & Both altered & $\mathrm{P}$ \\
\hline Total (\%) & $88(39)$ & $72(32)$ & & $108(48)$ & $44(20)$ & & $92(41)$ & $82(36)$ & \\
\hline \multicolumn{10}{|l|}{ Gender (\%) } \\
\hline Female & $19(41)$ & $16(35)$ & & $24(52)$ & $8(17)$ & & $19(41)$ & $20(43)$ & \\
\hline Male & $69(38)$ & $56(31)$ & 0.673 & $84(47)$ & $36(20)$ & 0.797 & $73(41)$ & $62(34)$ & 0.308 \\
\hline \multicolumn{10}{|l|}{ Pathologic tumor stage (\%) } \\
\hline pTa, pTis, or pT1 & $17(40)$ & $6(14)$ & & $14(33)$ & $6(14)$ & & $17(41)$ & 8 (19) & \\
\hline pT2 & $25(38)$ & $14(22)$ & & $30(46)$ & $7(11)$ & & $27(42)$ & $16(25)$ & \\
\hline pT3 & $32(39)$ & $35(42)$ & & $45(54)$ & $19(23)$ & & $34(41)$ & $40(48)$ & \\
\hline pT4 & $14(39)$ & $17(47)$ & $<0.001$ & $19(53)$ & $12(33)$ & $<0.001$ & $14(39)$ & $18(50)$ & $<0.001$ \\
\hline \multicolumn{10}{|l|}{ Pathological grade (\%) } \\
\hline Grade 1 or 2 & $9(53)$ & 0 & & $7(41)$ & 0 & & $8(47)$ & $1(6)$ & \\
\hline Grade 3 & $79(38)$ & $72(34)$ & 0.012 & $101(48)$ & $44(21)$ & 0.023 & $84(40)$ & $81(39)$ & 0.009 \\
\hline \multicolumn{10}{|l|}{ Lymph node status (\%) ${ }^{\mathrm{a}}$} \\
\hline No & $61(38)$ & $39(24)$ & & $72(45)$ & $23(14)$ & & $68(43)$ & $42(26)$ & \\
\hline $\mathrm{N} 1, \mathrm{~N} 2$ & $27(42)$ & $32(49)$ & $<0.001$ & $35(54)$ & $21(32)$ & $<0.001$ & $24(37)$ & $39(60)$ & $<0.001$ \\
\hline \multicolumn{10}{|c|}{ Lymphovascular invasion $(\%)^{\mathrm{a}}$} \\
\hline Negative & 47 (38) & $27(22)$ & & $53(43)$ & $17(14)$ & & $50(40)$ & $32(26)$ & \\
\hline Positive & $41(41)$ & $44(44)$ & $<0.001$ & $54(53)$ & $27(27)$ & $<0.001$ & $42(42)$ & 49 (49) & $<0.001$ \\
\hline \multicolumn{10}{|l|}{ Concomitant CIS $(\%)^{\mathrm{a}}$} \\
\hline Negative & $48(36)$ & $39(30)$ & & $66(50)$ & $20(15)$ & & $48(36)$ & $44(33)$ & \\
\hline Positive & $40(43)$ & $32(34)$ & 0.175 & $41(44)$ & $24(26)$ & 0.139 & $44(47)$ & $37(40)$ & 0.009 \\
\hline \multicolumn{10}{|l|}{ Stage grouping ${ }^{\mathrm{a}}$} \\
\hline Organ confined & $36(37)$ & $16(17)$ & & $39(40)$ & $9(9)$ & & $39(40)$ & $19(20)$ & \\
\hline Extra-vesical extension & $25(39)$ & $24(38)$ & & $34(53)$ & $14(22)$ & & $29(45)$ & $24(38)$ & \\
\hline \multirow[t]{2}{*}{ Lymph node metastases } & $27(42)$ & $32(49)$ & $<0.001$ & $35(54)$ & $21(32)$ & $<0.001$ & $24(37)$ & $39(60)$ & $<0.001$ \\
\hline & \multicolumn{3}{|c|}{$p R B$ and $p 21$ expression } & \multicolumn{3}{|c|}{$p R B$ and $p 27$ expression } & \multicolumn{3}{|c|}{ p21 and p27 expression } \\
\hline Total (\%) & $102(45)$ & $43(19)$ & & $88(39)$ & $80(35)$ & & $112(50)$ & $50(22)$ & \\
\hline \multicolumn{10}{|l|}{ Gender (\%) } \\
\hline Female & $29(63)$ & $6(13)$ & & $18(39)$ & $21(46)$ & & $27(59)$ & $11(24)$ & \\
\hline Male & $73(41)$ & $37(21)$ & 0.054 & $70(39)$ & $59(33)$ & 0.124 & $85(47)$ & $39(22)$ & 0.174 \\
\hline \multicolumn{10}{|l|}{ Pathologic tumor stage (\%) } \\
\hline pTa, pTis, or pT1 & $15(36)$ & $6(14)$ & & $14(33)$ & $10(24)$ & & $13(31)$ & $9(21)$ & \\
\hline pT2 & $25(39)$ & $9(14)$ & & $22(34)$ & $18(28)$ & & $33(51)$ & $8(12)$ & \\
\hline pT3 & $43(52)$ & $17(21)$ & & $38(46)$ & $35(42)$ & & $47(57)$ & $21(25)$ & \\
\hline pT4 & $19(53)$ & $11(31)$ & 0.010 & $14(39)$ & $17(47)$ & $<0.001$ & $19(53)$ & $12(33)$ & $<0.001$ \\
\hline \multicolumn{10}{|l|}{ Pathological grade (\%) } \\
\hline Grade 1 or 2 & $6(35)$ & $3(18)$ & & $7(41)$ & $4(24)$ & & $7(41)$ & $3(18)$ & \\
\hline Grade 3 & $96(46)$ & $40(19)$ & 0.589 & $81(39)$ & $76(36)$ & 0.492 & $105(50)$ & $47(23)$ & 0.472 \\
\hline \multicolumn{10}{|l|}{ Lymph node status (\%) ${ }^{\mathrm{a}}$} \\
\hline No & $65(41)$ & $29(18)$ & & $59(37)$ & $49(31)$ & & $76(48)$ & $30(19)$ & \\
\hline $\mathrm{N} 1, \mathrm{~N} 2$ & $36(55)$ & $14(22)$ & 0.034 & $29(45)$ & $30(46)$ & 0.001 & $35(54)$ & $20(31)$ & 0.012 \\
\hline Lymphovascular invasion ( & $\%)^{\mathrm{a}}$ & & & & & & & & \\
\hline Negative & $48(38)$ & $21(17)$ & & $39(32)$ & $39(32)$ & & $51(41)$ & $26(21)$ & \\
\hline Positive & $53(53)$ & $22(22)$ & 0.015 & $49(49)$ & $40(40)$ & $<0.001$ & $60(59)$ & $24(24)$ & 0.002 \\
\hline Concomitant CIS (\%) ${ }^{\mathrm{a}}$ & & & & & & & & & \\
\hline Negative & $54(41)$ & $28(21)$ & & $42(32)$ & $49(37)$ & & $64(49)$ & $28(21)$ & \\
\hline Positive & $47(51)$ & $15(16)$ & 0.337 & $46(50)$ & $30(32)$ & 0.017 & $47(51)$ & $22(24)$ & 0.748 \\
\hline Stage grouping & & & & & & & & & \\
\hline Organ-confined & $35(36)$ & $13(13)$ & & $31(32)$ & $25(26)$ & & $42(43)$ & $14(14)$ & \\
\hline Extra-vesical extension & $31(48)$ & $16(25)$ & & $28(44)$ & $25(39)$ & & $35(55)$ & $16(25)$ & \\
\hline Lymph node metastases & $36(55)$ & $14(22)$ & 0.002 & $29(45)$ & $30(46)$ & 0.001 & $35(54)$ & $20(31)$ & 0.001 \\
\hline
\end{tabular}

${ }^{\mathrm{a}}$ Lymph node status, lymphovascular invasion, and concomitant carcinoma in situ were not available in one patient. 
Table 5 Multivariable Cox regression analyses of pathologic features and molecular markers for the prediction of disease recurrence and disease-specific mortality in 226 consecutive patients treated with radical cystectomy for urothelial cell carcinoma of the bladder

\begin{tabular}{|c|c|c|c|c|c|c|}
\hline & \multicolumn{3}{|c|}{ Recurrence } & \multicolumn{3}{|c|}{ Bladder cancer-specific mortality } \\
\hline & Risk ratio & $95 \% C I$ & $\mathrm{P}$ & Risk ratio & $95 \% C I$ & $P$ \\
\hline \multicolumn{7}{|l|}{ Model 1} \\
\hline Pathologic tumor grade & 1.219 & $0.366-4.050$ & 0.746 & 2.873 & $0.384-21.518$ & 0.304 \\
\hline \multicolumn{7}{|l|}{ Pathologic tumor stage } \\
\hline Organ confined ( $\leq \mathrm{pT} 3 \mathrm{~N}$ ) & 1.000 & Referent & - & 1.000 & Referent & - \\
\hline Extravesical extension ( $\geq$ pT3 N0) & 3.536 & $1.840-6.794$ & $<0.001$ & 4.094 & $1.942-8.629$ & $<0.001$ \\
\hline Lymph node metastases (pTany Npositive) & 5.791 & $2.777-12.073$ & $<0.001$ & 7.517 & $3.270-17.278$ & $<0.001$ \\
\hline Test for trend & & & $<0.001$ & & & $<0.001$ \\
\hline Lymphovascular invasion & 1.461 & $0.915-2.331$ & 0.113 & 1.374 & $0.825-2.290$ & 0.222 \\
\hline Adjuvant chemotherapy & 1.065 & $0.664-1.700$ & 0.794 & 0.576 & $0.340-0.976$ & 0.040 \\
\hline \multicolumn{7}{|l|}{ Combined p53 and p21 status } \\
\hline Both wild & 1.000 & Referent & - & 1.000 & Referent & - \\
\hline One altered & 1.548 & $1.008-2.703$ & 0.046 & 2.726 & $1.308-5.683$ & 0.002 \\
\hline Both altered & 2.094 & $1.116-3.929$ & 0.021 & 3.488 & $1.566-7.766$ & 0.007 \\
\hline Test for trend & & & 0.028 & & & 0.009 \\
\hline \multicolumn{7}{|l|}{ Model 2} \\
\hline Pathologic tumor grade & 1.157 & $0.346-3.869$ & 0.813 & 2.439 & $0.322-18.479$ & 0.388 \\
\hline \multicolumn{7}{|l|}{ Pathologic tumor stage } \\
\hline Organ confined ( $\leq \mathrm{pT} 3 \mathrm{~N}$ ) & 1.000 & Referent & - & 1.000 & Referent & - \\
\hline Extravesical extension ( $\geq$ pT3 No) & 3.088 & $1.612-5.915$ & $<0.001$ & 3.984 & $1.887-8.415$ & $<0.001$ \\
\hline Lymph node metastases (pTany Npositive) & 5.137 & $2.510-10.516$ & $<0.001$ & 6.968 & $3.089-15.719$ & $<0.001$ \\
\hline Test for trend & & & $<0.001$ & & & $<0.001$ \\
\hline Lymphovascular invasion & 1.391 & $0.880-2.198$ & 0.158 & 1.367 & $0.831-2.248$ & 0.218 \\
\hline Adjuvant chemotherapy & 1.019 & $0.632-1.643$ & 0.937 & 0.538 & $0.317-0.912$ & 0.021 \\
\hline \multicolumn{7}{|l|}{ Number of altered markers } \\
\hline None & 1.000 & Referent & - & 1.000 & Referent & - \\
\hline One & 4.970 & $0.643-38.382$ & 0.100 & 2.363 & $0.288-19.350$ & 0.423 \\
\hline Two & 7.426 & $0.973-56.683$ & 0.053 & 5.873 & $0.758-45.521$ & 0.090 \\
\hline Three & 8.926 & $1.167-68.261$ & 0.035 & 6.566 & $1.043-51.134$ & 0.041 \\
\hline Four & 9.597 & $1.189-77.480$ & 0.034 & 9.370 & $1.142-76.898$ & 0.033 \\
\hline Test for trend & & & 0.042 & & & 0.046 \\
\hline
\end{tabular}

\section{Correlation of p53, pRB, p21, and p27 Expression between Matched Transurethral Resection and Radical Cystectomy Specimens}

Twenty-two patients with non-muscle invasive disease underwent early radical cystectomy for grade III T1 disease and/or non-muscle invasive disease refractory to intra-vesical chemotherapy/ immunotherapy. Concordance between marker expression in transurethral resection and cystectomy specimens was $82,86,82$ and $77 \%$ for p53, pRB, p21 and p27, respectively. These rates were statistically significant in all cases (all $P$-values $\leq 0.030$ ).

\section{Correlation of p53, pRB, p21 and p27 Expression between Matched Radical Cystectomy and Metastatic Lymph Node Specimens}

We also stained the lymph nodes with urothelial carcinoma from 50 of the 65 patients who had metastases to lymph nodes for p53, pRB, p21 and p27. Concordance between marker expression in the lymph nodes and matched radical cystectomy specimens was $92,82,70$ and $86 \%$ for p53, pRB, p21 and p27, respectively. These rates were statistically significant in all cases $(P$-values $\leq 0.009)$.

\section{Discussion}

The clinical usefulness of biomarkers in bladder cancer in addition to the well-known pathological parameters such as tumor grade and stage has been a subject of controversy given the discrepancy between various studies. The failure to detect mutations or aberrant expression at one point of a biological pathway does not necessarily rule out defects further downstream. Our study highlights the need to investigate multiple steps of a pathway or multiple different pathways before determining if the pathway may or may not be involved in cancer progression. It also stresses that multiple biomarkers determination rather than single markers are way more useful as prognostic factors. 
Table 6 Associations of number of altered biomarkers status in the primary cancer with clinical and pathologic characteristics of 226 consecutive patients treated with radical cystectomy for urothelial cell carcinoma of the bladder

\begin{tabular}{|c|c|c|c|c|c|c|}
\hline & \multicolumn{6}{|c|}{ Number of altered biomarkers in primary cancer $(\mathrm{n}=226)$} \\
\hline & None altered & One altered & Two altered & Three altered & Four altered & $\mathrm{P}$ \\
\hline Total (\%) & $28(12)$ & $56(25)$ & $59(26)$ & $62(27)$ & $21(9)$ & \\
\hline \multicolumn{7}{|l|}{ Gender (\%) } \\
\hline Female & $2(4)$ & $11(24)$ & $13(28)$ & $17(37)$ & $3(7)$ & \\
\hline Male & $26(14)$ & $45(25)$ & $46(26)$ & $45(25)$ & $18(10)$ & 0.238 \\
\hline \multicolumn{7}{|l|}{ Pathologic tumor stage (\%) } \\
\hline pTa, pTis, or pT1 & $12(29)$ & $13(31)$ & $6(14)$ & $9(21)$ & $2(5)$ & \\
\hline pT2 & $14(22)$ & $18(28)$ & $18(28)$ & $12(19)$ & $3(5)$ & \\
\hline pT3 & $2(2)$ & $18(22)$ & $26(31)$ & $27(33)$ & $10(12)$ & \\
\hline pT4 & 0 & 7 (19) & $9(25)$ & $14(39)$ & $6(17)$ & $<0.001$ \\
\hline \multicolumn{7}{|l|}{ Pathological grade (\%) } \\
\hline Grade 1 or 2 & $4(24)$ & $6(35)$ & $5(29)$ & $2(12)$ & 0 & \\
\hline Grade 3 & $24(12)$ & $50(24)$ & $54(26)$ & $60(29)$ & $21(10)$ & 0.192 \\
\hline \multicolumn{7}{|l|}{ Lymph node status $(\%)^{a}$} \\
\hline No & $28(18)$ & $47(29)$ & $38(24)$ & $36(23)$ & $11(7)$ & \\
\hline $\mathrm{N} 1, \mathrm{~N} 2$ & 0 & $9(14)$ & $21(32.3)$ & $25(39)$ & $10(15)$ & $<0.001$ \\
\hline \multicolumn{7}{|l|}{ Lymphovascular invasion $(\%)^{a}$} \\
\hline Negative & $26(21)$ & $38(31)$ & $24(19)$ & $26(21)$ & $10(8)$ & \\
\hline Positive & $2(2)$ & $18(18)$ & $35(35)$ & $35(35)$ & $11(11)$ & $<0.001$ \\
\hline \multicolumn{7}{|l|}{ Concomitant carcinoma in situ $(\%)^{\mathrm{a}}$} \\
\hline Negative & $21(16)$ & $35(27)$ & $31(24)$ & $31(24)$ & $14(11)$ & \\
\hline Positive & $7(8)$ & $21(23)$ & $28(30)$ & $30(32)$ & $7(8)$ & 0.169 \\
\hline \multicolumn{7}{|l|}{ Stage grouping $(\%)^{a}$} \\
\hline Organ-confined $(<\mathrm{pT} 3 \mathrm{~N} 0)$ & $26(27)$ & $29(30)$ & $21(22)$ & $17(18)$ & $4(4)$ & \\
\hline Extra-vesical extension ( $\geq$ pT3 No) & $2(3)$ & $18(28)$ & $17(27)$ & $20(31)$ & $7(11)$ & \\
\hline Lymph node metastases (pTany N positive) & 0 & $9(14)$ & $21(32)$ & $25(39)$ & $10(15)$ & $<0.001$ \\
\hline
\end{tabular}

${ }^{\mathrm{a}}$ Lymph node status, lymphovascular invasion, and concomitant carcinoma in situ were not available in one patient.

First, we confirmed that altered expression of p53, pRB, p21 and p27 is associated with established features of aggressive primary bladder cancer, lymph node metastases and eventual disease progression and death of bladder cancer., ${ }^{4,5} 7-10,12-14$ Although all four biomarkers were independently associated with bladder cancer outcomes when evaluated separately, we identified definite distinctions in the associations of p53, pRB, p21 and p27 with pathologic features of the local tumor and disease prognosis. For example, the percent specimens with altered status of p53, pRB or p27 was lowest in normal urothelium areas within cystectomy specimens, and increased gradually from non-muscle invasive urothelial carcinoma obtained by transurethral resection, to concomitant carcinoma in situ areas within cystectomy specimens, to primary cancer within cystectomy specimens, and was highest in metastatic lymph node specimens. Conversely, p21 expression, a downstream effector of p53, was not different between non-muscle invasive urothelial carcinoma, concomitant carcinoma in situ areas within cystectomy specimens, and primary cancer within cystectomy specimens. Similarly, whereas p53, pRB and p27 were all three associated with advanced pathologic stage, lymphovascular invasion and lymph node metastases, p21 was not. A characteristic specific to p53 was that it was the sole biomarker associated with features of cellular dedifferentiation (ie, tumor grade) and concomitant carcinoma in situ, which is an aggressive precursor lesion of invasive tumors. ${ }^{16,19,21-23}$ These differences in the proportion of alterations along the progression model may reflect the role of each cell-cycle regulator and their cumulative effect in carcinogenesis.

We found that p53, p21 and p27, but not pRB, were independently associated with disease recurrence and bladder cancer-specific survival in the subgroup of patients with organ-confined disease. These findings suggest that molecular staging of human bladder cancer by p53, p21 and/or p27 immunohistochemistry could be potentially useful in selecting appropriate treatment strategy and timing for patients with high probability of developing disease progression despite apparent effective local therapy. The evaluation of several biomarkers by immunohistochemistry may help overcome some 

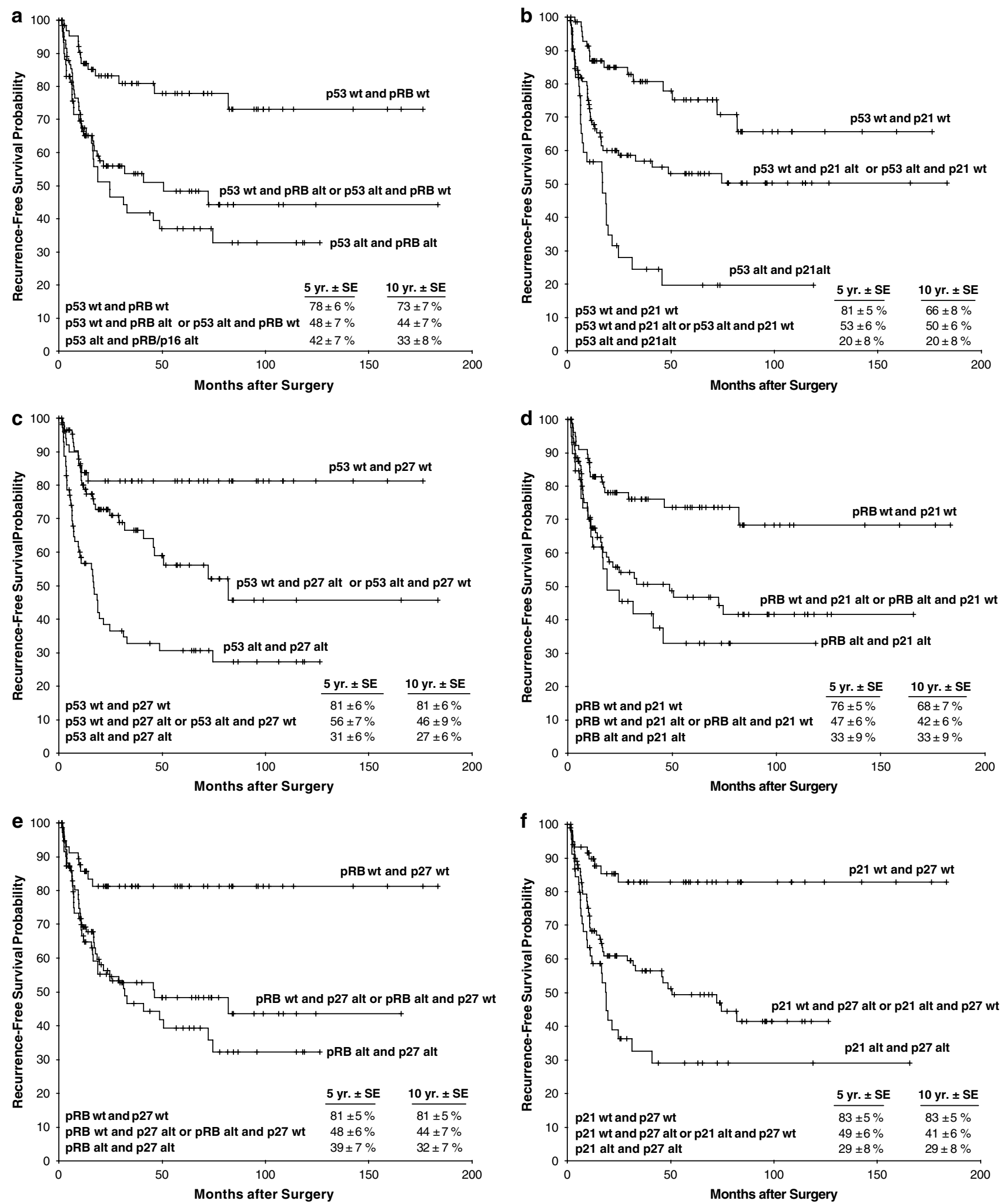

Figure 1 Probability of bladder cancer recurrence-free survival in 226 patients treated with radical cystectomy for urothelial cell carcinoma of the bladder according to (a) the combined p53 and pRB status; (b) the combined p53 and p21 status; (c) the combined p53 and p27 status; (d) the combined pRB and p21 status; (e) the combined pRB and p27 status; (f) the combined p21 and p27 status. 

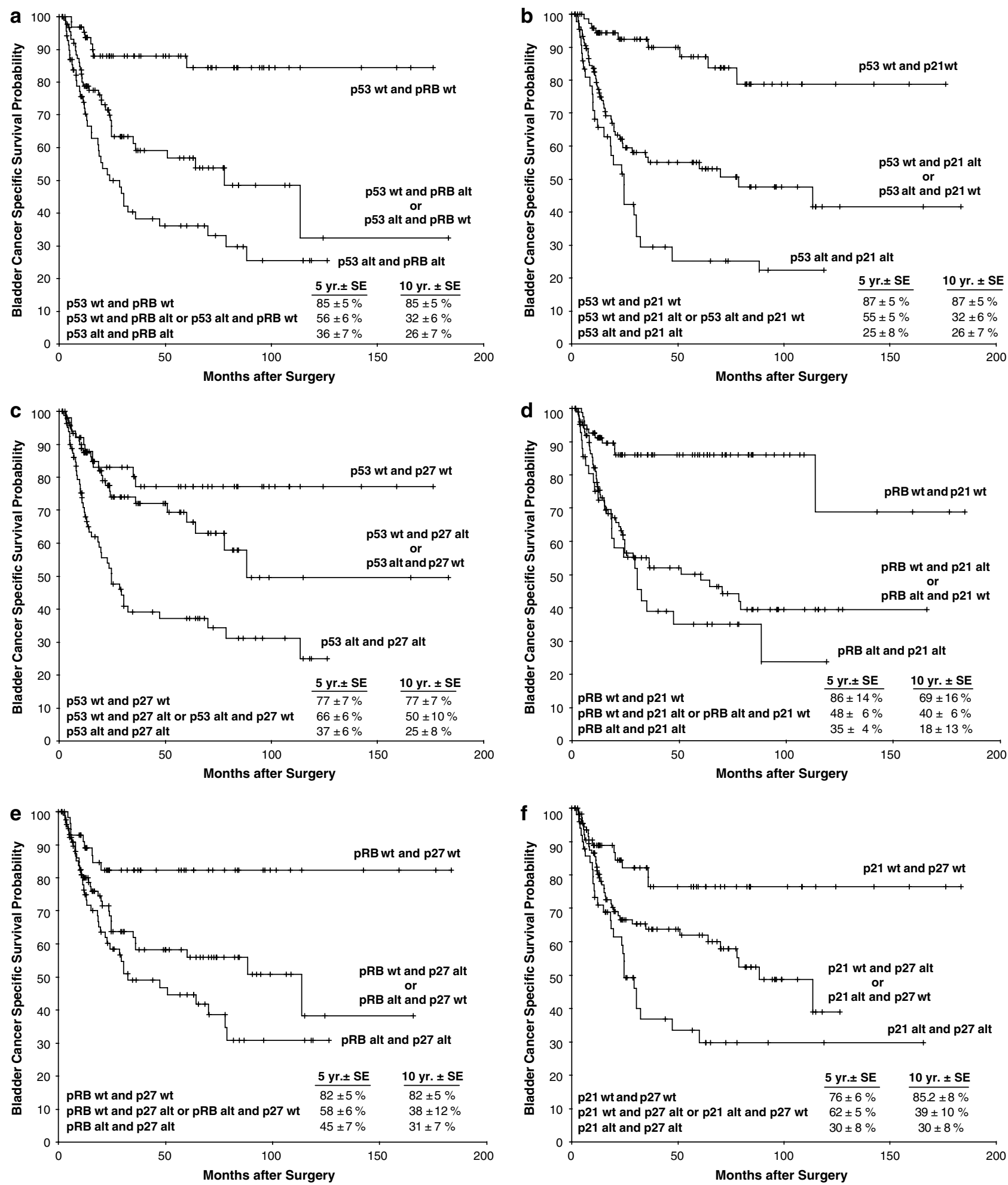

Figure 2 Probability of bladder cancer-specific survival in 226 patients treated with radical cystectomy for urothelial cell carcinoma of the bladder according to (a) the combined p53 and pRB status; (b) the combined p53 and p21 status; (c) the combined p53 and p27 status; (d) the combined pRB and p21 status; (e) the combined pRB and p27 status; (f) the combined p21 and p27 status.

of the limitations found when analyzing single markers with this technique as there may be significant discrepancy between tissue expression and biological activity.
We found that p27 expression was strongly associated with bladder cancer presence, progression, metastasis and mortality. p27 is a key regulator of progression from $\mathrm{G} 1$ to $\mathrm{S}$ phase, where it can 

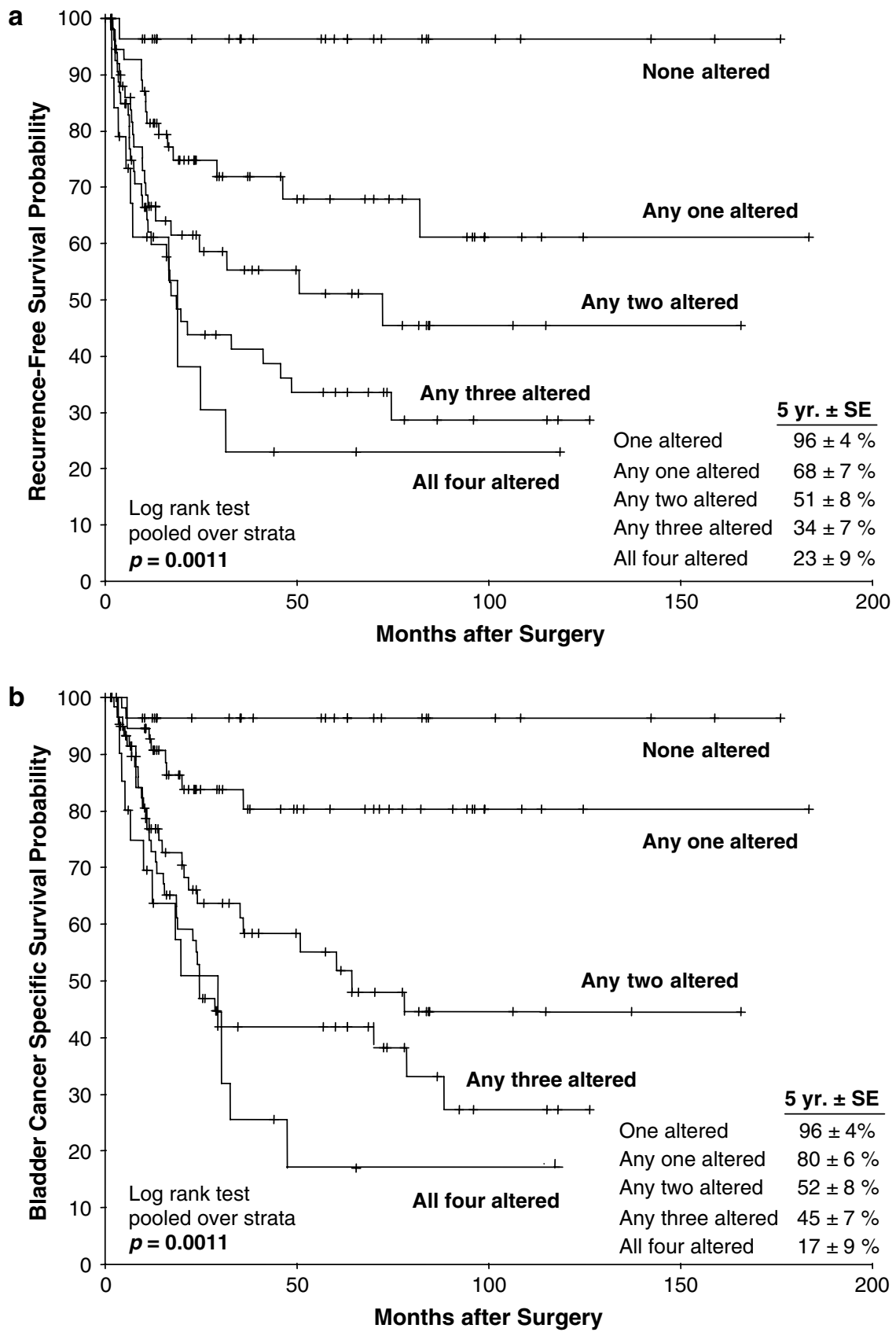

Figure 3 Probability of (a) bladder cancer recurrence-free survival and (b) bladder cancer-specific survival in 226 patients treated with radical cystectomy for urothelial cell carcinoma of the bladder according to the number of altered markers.

induce a block in the cell cycle. Therefore, defective regulation at the p27 checkpoint can result in uncontrolled cellular proliferation. In the present study, as one would have expected it, p27 expression was normal in all patients with normal urothelium and altered in $47 \%$ of patients with non-muscle invasive tumor stage suggesting that it contributes to the early stages of bladder carcinogenesis. Alteration of p27 expression increased with more advanced stage and was an independent predictor of disease recurrence and bladder cancerspecific mortality in patients with treated with radical cystectomy. To our knowledge, only one study has evaluated the association of p27 expression with outcomes of patients treated with radical cystectomy to date. When evaluated in 26 patients treated with radical cystectomy and 23 patients treated with transurethral resection, Del Pizzo et $a l^{24}$ reported that $\mathrm{p} 27$ expression progressively decreased with increasing tumor stage and was associated with increased all cause mortality in univariate analysis.

This study demonstrates that analysis of p53, pRB, p21 and p27 in combination provide addi- 
tional prognostic information beyond that obtained from any single biomarker or combination of two biomarkers. This makes sense because it investigates multiple pathways including downstream effector pathways rather than a single crossroad in a pathway. Higher total number of altered biomarkers was associated with a progressive, proportional increase in the risk of advanced pathologic tumor stage, lymphovascular invasion, lymph node metastases, disease recurrence and death of bladder cancer. The expression of cell-cycle regulators in urothelial carcinoma was commonly altered in the present study suggesting a role in carcinogenesis. However, the rate of altered expression of at least one biomarker and, more importantly, the number of altered biomarkers both increased progressively with advancing pathologic stages and presence of features of biologically aggressive disease such as lymphovascular invasion and metastases to lymph nodes. Furthermore, the higher the number of biomarkers with altered expression, the higher the risk of recurrence and bladder cancer-related death, after adjusting for the effects of standard pathologic features. The majority of patients with bladder cancer showing alterations in all four biomarkers will suffer from tumor recurrence and eventually die of their disease, no matter what the stage of disease at presentation (ie, pathologic stage and lymph node status). Moreover, patients with bladder cancers that show no evidence of alterations in any of the four cell-cycle regulators harbor very low risks of recurrence, regardless of disease stage. Thus, knowledge of the status of all four cell-cycle regulators may provide more specific information about likely disease outcome. Because tumorigenesis and progression is a process involving multiple genetic defects, it is likely that alterations in other pathways will also affect bladder cancer progression and metastasis. These data, together with the current paradigm that bladder cancer develops along multiple molecular pathways, suggest that including multiple biomarkers in models designed to predict outcomes following cystectomy could enhance the predictive power.

We found that transurethral resection specimens accurately reflect staining patterns of p53, pRB, p21 and p27 in matched cystectomy specimens with concordance rates ranging from 77 to $86 \%$. This together with the finding that altered expression of these four biomarkers are independently associated with an increased risk of bladder cancer progression in patients with non-muscle invasive urothelial carcinoma ${ }^{18}$ suggests that risk assessment based on molecular marker status can be performed on biopsy specimens before definitive therapy. This information is of interest as assessment of disease aggressiveness could prove useful before embarking on a defined therapy. This assessment could be potentially useful in prospective studies of neo-adjuvant chemotherapy or bladder sparing protocols. Although this is an improvement over current clinical prediction, assessment of biological markers on transurethral resection specimens carries the risk of being fraught with a close to $20 \%$ inaccurate estimation. Clinicians have to be aware of this discrepancy before decision-making.

This study has several limitations. First and foremost are the limitations inherent to any retrospective data collection and the reliability of immunohistochemical techniques. The sample size and relatively short follow-up may have limited our ability to detect small differences attributed to other variables. Immunohistochemistry is semi-quantitative and highly dependent on a range of poorly controlled variables such as choice of antibody, antibody concentration, fixation techniques, variability in the interpretation and stratification criteria, and inconsistency in specimen handling and technical procedures. To reduce the number of variables in immunohistochemistry analysis, we have chosen to use tissue microarrays, automated autostainer and automated scoring system based on bright field microscopy imaging coupled with advanced color detection software. ${ }^{25-27}$

In conclusion, p53, p21, p27 and pRB have a cooperative/synergistic role in the biologic behavior of urothelial carcinoma. An increasing number of altered molecular markers improve prediction of disease recurrence and survival in patients treated with radical cystectomy. Therefore, multiple molecular markers should be used in risk stratification, and may help identify patients who will benefit from adjuvant therapies. Predictive tools such as nomograms or artificial neuronal networks combining various biomarker based on large series of patients may help improve our ability to prognosticate disease outcomes and counsel patients with more accuracy.

\section{References}

1 Levine AJ, Momand J, Finlay CA. The p53 tumour suppressor gene. Nature 1991;351:453-456.

2 Hollstein M, Sidransky D, Vogelstein B, et al. p53 mutations in human cancers. Science 1991;253:49-53.

3 Ruas M, Peters G. The p16INK4a/CDKN2A tumor suppressor and its relatives. Biochim Biophys Acta 1998;1378:F115-F177.

4 Esrig D, Spruck CHD, Nichols PW, et al. p53 nuclear protein accumulation correlates with mutations in the p53 gene, tumor grade, and stage in bladder cancer. Am J Pathol 1993;143:1389-1397.

5 Cote RJ, Dunn MD, Chatterjee SJ, et al. Elevated and absent $\mathrm{pRb}$ expression is associated with bladder cancer progression and has cooperative effects with p53. Cancer Res 1998;58:1090-1094.

6 Grossman HB, Liebert M, Antelo M, et al. p53 and RB expression predict progression in T1 bladder cancer. Clin Cancer Res 1998;4:829-834.

7 Sarkis AS, Dalbagni G, Cordon-Cardo C, et al. Nuclear overexpression of p53 protein in transitional cell bladder carcinoma: a marker for disease progression. J Natl Cancer Inst 1993;85:53-59. 
8 Esrig D, Elmajian D, Groshen S, et al. Accumulation of nuclear p53 and tumor progression in bladder cancer. N Engl J Med 1994;331:1259-1264.

9 Stein JP, Ginsberg DA, Grossfeld GD, et al. Effect of p21WAF1/CIP1 expression on tumor progression in bladder cancer. J Natl Cancer Inst 1998;90:1072-1079.

10 Tokunaga H, Shariat SF, Green AE, et al. Correlation of immunohistochemical molecular staging of bladder biopsies and radical cystectomy specimens. Int J Radiat Oncol Biol Phys 2001;51:16-22.

11 Benedict WF, Lerner SP, Zhou J, et al. Level of retinoblastoma protein expression correlates with p16 (MTS-1/INK4A/CDKN2) status in bladder cancer. Oncogene 1999;18:1197-1203.

12 Cordon-Cardo C, Wartinger D, Petrylak D, et al. Altered expression of the retinoblastoma gene product: prognostic indicator in bladder cancer. J Natl Cancer Inst 1992;84:1251-1256.

13 Shariat SF, Tokunaga H, Zhou J, et al. p53, p21, pRB, and p16 expression predict clinical outcome in cystectomy with bladder cancer. J Clin Oncol 2004; 22:1014-1024.

14 Chatterjee SJ, Datar R, Youssefzadeh D, et al. Combined effects of p53, p21, and $\mathrm{pRb}$ expression in the progression of bladder transitional cell carcinoma. J Clin Oncol 2004;22:1007-1013.

15 Cordon-Cardo C, Zhang ZF, Dalbagni G, et al. Cooperative effects of p53 and pRB alterations in primary superficial bladder tumors. Cancer Res 1997; 57:1217-1221.

16 Shariat SF, Kim J, Raptidis G, et al. Association of p53 and p21 expression with clinical outcome in patients with carcinoma in situ of the urinary bladder. Urology 2003;61:1140-1145.

17 Shariat SF, Weizer AZ, Green A, et al. Prognostic value of P53 nuclear accumulation and histopathologic features in $\mathrm{T} 1$ transitional cell carcinoma of the urinary bladder. Urology 2000;56:735-740.

18 Shariat SF, Ashfaq R, Sagalowsky AI, et al. Predictive value of cell cycle biomarkers in non-muscle invasive bladder transitional cell carcinoma. J Uro 2007;177: 481-487.

19 Cordon-Cardo C, Dalbagni G, Saez GT, et al. p53 mutations in human bladder cancer: genotypic vs phenotypic patterns. Int J Cancer 1994;56:347-353.

20 Shariat SF, Karakiewicz PI, Palapattu GS, et al. Outcomes of radical cystectomy for transitional cell carcinoma of the bladder: a contemporary series from the bladder cancer research consortium. J Urol 2006; 176:2414-2422.

21 Spruck III CH, Ohneseit PF, Gonzalez-Zulueta M, et al. Two molecular pathways to transitional cell carcinoma of the bladder. Cancer Res 1994;54:784-788.

22 Hartmann A, Schlake G, Zaak D, et al. Occurrence of chromosome 9 and p53 alterations in multifocal dysplasia and carcinoma in situ of human urinary bladder. Cancer Res 2002;62:809-818.

23 Wagner U, Sauter G, Moch H, et al. Patterns of p53, erbB-2, and EGF-r expression in premalignant lesions of the urinary bladder. Hum Pathol 1995;26:970-978.

24 Del Pizzo JJ, Borkowski A, Jacobs SC, et al. Loss of cell cycle regulators p27(Kip1) and cyclin E in transitional cell carcinoma of the bladder correlates with tumor grade and patient survival. Am J Pathol 1999;155: 1129-1136.

25 Divi RL, Dragan YP, Pitot HC, et al. Immunohistochemical localization and semi-quantitation of hepatic
tamoxifen-DNA adducts in rats exposed orally to tamoxifen. Carcinogenesis 2001;22:1693-1699.

26 Wang S, Saboorian MH, Frenkel EP, et al. Assessment of HER-2/neu status in breast cancer. Automated Cellular Imaging System (ACIS)-assisted quantitation of immunohistochemical assay achieves high accuracy in comparison with fluorescence in situ hybridization assay as the standard. Am J Clin Pathol 2001;116: 495-503.

27 Bauer KD, de la Torre-Bueno J, Diel IJ, et al. Reliable and sensitive analysis of occult bone marrow metastases using automated cellular imaging. Clin Cancer Res 2000;6:3552-3559.

\section{Appendix A}

\section{Tissue Microarray}

The tissue microarrays comprised (1) tumor tissue from the primary, index cancer from 74 patients treated with TUR; (2) tumor tissue from the primary, index cancer from 226 patients who were treated with radical cystectomy; (3) histologically tumorfree tissue from areas adjacent to primary TCC from 72 of the 226 patients who were treated with radical cystectomy; (4) CIS tissue from areas adjacent to the primary, index cancer from 40 patients who were treated with radical cystectomy; (5) lymph node tissue involved with cancer from 50 of the 226 patients who were treated with radical cystectomy; and (6) non-cancer tissue from nine patients who were treated with cystectomy for other causes than malignancy without evidence of any cancer.

Tissue blocks were stored under ambient conditions, at approximately $24^{\circ} \mathrm{C}$. H\&E-stained sections were reviewed to select representative areas of the index tumor from which to acquire cores for microarray analysis. The index tumor was defined as the largest and/or highest tumor stage and grade. Tissue microarray blocks were constructed by taking core samples from morphologically representative areas of paraffin-embedded tumor tissues and assembling them on a recipient paraffin block. This was performed with a precision instrument (Beecher Instruments, Silver Spring, MD, USA) that uses two separate core needles for punching the donor and recipient blocks and a micrometer-precise coordinate system for assembling tissue samples on a block. For each case, three replicate $0.6 \mathrm{~mm}$ core diameter samples were collected and placed on separate recipient blocks. All samples were spaced $0.5 \mathrm{~mm}$ apart. Multiple sausage internal controls were also placed with the standard controls. Fivemicrometer sections were obtained from the microarray and stained with H\&E to confirm the presence of tumor and to assess the tumor histology. Tumor samples were randomly arranged on the blocks.

Sample tracking was based on coordinate positions for each tissue spot in the tissue microarray block; the spots were transferred onto tissue microarray slides for staining. This sample tracking system was linked to a Microsoft Excel database 
containing demographic, clinical, pathologic, and survival data on each patient. The array was read according to the given tissue microarray map, each core was scored individually, and the results were presented as the mean of the three replicate core samples.

\section{Appendix B}

\section{Immunohistochemical Scoring}

Nuclear p53 immunoreactivity was considered altered when samples demonstrated at least $10 \%$ nuclear reactivity, because it has been shown that accumulation of p53 protein in $10 \%$ or more of the tumor-cell nuclei strongly correlates with mutations in the p53 gene. ${ }^{6,21}$ Tumors were classified as p21-altered consisting of those tumors with no detectable or only very low levels of p21 nuclear immunoreactivity $(0-9.9 \%$ of tumor cells showing p21 expression, p21 altered status) or p21 normal consisting of those tumors with moderate to high levels of p21 nuclear immunoreactivity $(10 \%$ of tumor cells showing p21 expression). pRB immunoreactivity was assigned to one of three categories of nuclear staining in the tumor cells: no nuclear reactivity; normal heterogeneous; and strong homogeneous (more than $50 \%)$. Tumors with no pRB expression and those with a strong homogeneous staining pattern were categorized as having altered pRB status, as it has been shown that an overexpression of $\mathrm{pRB}$ in bladder cancer is also indicative of dysfunctional pRB status. $^{13,15}$ Nuclear p27 immunoreactivity was considered altered when samples demonstrated less than $30 \%$ nuclear reactivity, because this level was previously determined to be clinically relevant. ${ }^{20}$ 